\title{
INFORMATION, INSTITUTIONS ET TEMPORALITÉ QUELQUES REMARQUES CRITIQUES SUR L'USAGE DE LA NOUVELLE ÉCONOMIE DE L'INFORMATION EN HISTOIRE
}

\author{
Alessandro StANZIANI
}

Résumé : L'article fait le point sur les thèses néo-institutionnalistes et sur la théorie des jeux telles qu'elles ont été « appliquées » à l'histoire. L'analyse des marchés principaux (travail, terre, crédit) et leur impact sur la théorie du développement sont également présentés. L'auteur prend ensuite en considération la théorie des jeux, il en discute les hypothèses et les présupposés de manière à rendre explicites les usages qui en ont été faits en histoire. La dernière partie introduit des notions plus complexes de rationalité économique, distingue entre information et connaissance et propose un schéma heuristique permettant d'intégrer certains éléments de l'économie de l'information et des jeux dans des démarches qui tiennent compte des notions temporelles propres à l'historiographie.

Mots-CLÉs : information, institution, théorie des jeux, marché.

ABSTRACT: The paper reviews the main "applications » to economic history of neo-institutionalist and game theories. The analysis of labor, land and credit markets as well as their implications for the theory of development are equally discussed. The author then discusses the main game theories, their hypotheses and assertions in order to elucidate their historiographical uses. The last part of the paper introduces more complex notions of rationality, while distinguishing between information and learning; it finally suggests an heuristic scheme able to integrate certain elements of the economy of information and games into historical notions of time.

KEYWORDS : information, institution, game theory, market.

Revue de synthèse : $4^{\mathrm{e}} \mathrm{S} . \mathrm{n}^{\mathrm{os}} 1-2$, janv.-juin 2000, p. 117-155. 
Zusammenfassung : Der Artikel behandelt die wichtigsten Anwendungen der neoinstitutionalistischen Theorien und der Spieltheorie auf die Wirtschaftsgeschichte. Ferner geht es um die Analyse der wichtigsten Märkte (Arbeit, Grund und Boden, Finanzen). Danach wird die Spieltheorie untersucht, wobei der Verfasser deren Annahmen und Voraussetzungen diskutiert, um ihre Anwendung in der Geschichtswissenschaft zu verdeutlichen. Im letzten Teil des Artikels werden komplexere Begriffe der wirtschaftlichen Rationalität eingeführt, wobei zwischen Information und Wissen unterschieden wird. Das dabei eingeführte heuristische Schema ermöglicht die Einbeziehung bestimmter Elemente der Informationsökonomie und der Spieltheorie bei der Untersuchung der in der Geschichtsschreibung verwendeten Zeitbegriffe.

STICHWÖRTER : Information, Institution, Spieltheorie, Markt.

RESUMEN : El articulo estudia la « aplicación » de las tesis neoinstitucionalistas y de la teoría de juegos a la historia. Se discute inicialmente el análisis de algunos mercados importantes (trabajo, tierra, crédito), y su impacto sobre la teoría del desarrollo. A continuación, se tratan las hipótesis y presupuestos de la teoría de juegos con objeto de mostrar el uso que se hace de ellos en historiografía. La parte final introduce concepciones más complejas de la racionalidad económica. Se distingue entre información y conocimiento, y se propone un esquema heurístico que permite integrar ciertos elementos de la economía de la información y de la teoría de juegos en enfoques teóricos que tengan en cuenta los conceptos temporales característicos de la historia.

PalabRas CLAVES : información, institución, teoría de juegos, mercado.

Alessandro StanZiani, né en 1961, est chargé de recherche au Centre national de la recherche scientifique. Ses travaux portent, d'une part, sur l'histoire de l'économie, de la pensée économique et statistique en Russie entre 1861 et 1930, et, d'autre part, sur le thème « histoire économique, information et institutions : les marchés du vin français et internationaux entre 1870 et $1914 »$.

Adresse : CNRS-IDHE, ENS Cachan, bât. Laplace, 61 av. du Président-Wilson, F-94235 Cachan.

Courrier électronique : stanzian@idhe.ens-cachan.fr 
Appliquée à l'art militaire d'abord, à l'analyse économique ensuite, la théorie des jeux a commencé, ces dernières années, à être aussi utilisée en histoire. L'analyse des stratégies et des décisions individuelles, celle des contrats et des rapports hiérarchiques, le fonctionnement des organisations ont été étudiés, afin de rendre compte de l'évolution du commerce, de l'organisation du travail, des rapports du crédit, etc. Ces démarches ont envisagé une échelle temporelle assez longue, s'étalant du Moyen Âge à nos jours.

Ces approches nouvelles s'inscrivent en réalité dans un courant historiographique plus important, qui a débuté au tournant des années 1960 et 1970, à savoir l'analyse des institutions et le rôle de l'information dans l'activité économique. En effet, les institutions et l'information économique «constituent les deux faces de la même monnaie ${ }^{1}$ ». Tout d'abord parce que les institutions commandent la production des informations économiques : que l'on songe à la production des statistiques officielles à partir du XVII siècle. Ce lien devient plus évident encore si, selon une perspective plus large, nous entendons par institutions les entreprises, les corporations et, de manière générale, un ensemble de règles conditionnant l'activité économique. Ainsi, les entreprises ont besoin d'informations, non seulement sur les marchés extérieurs, mais également sur l'activité de leur propre personnel. De même, les rapports de crédit exigent l'accumulation d'informations de la part des créanciers ; finalement, sur le marché du travail, la circulation des informations est importante tant du côté de l'offre (il suffit de rappeler le rôle joué par les réseaux familiaux, les coopératives, les syndicats) que de celui de la demande (bureaux d'intermédiaires, réseaux des entrepreneurs, informations au niveau du village, etc.).

Pourquoi s'interroger sur ces courants économiques et historiographiques? L'histoire des faits économiques se trouve aujourd'hui à un carrefour important : après les critiques apportées à l'encontre des études purement descriptives, d'une part, et à celle de la cliométrie, d'autre part, la théorie néo-institutionnaliste et, plus récemment, la théorie des jeux apparaissent comme des alternatives solides, surtout dans les pays anglo-saxons. En France, si la cliométrie n'a jamais vraiment décollé, les courants néoinstitutionnalistes, voire la théorie des jeux, sont encore envisagés avec un certain scepticisme. Ne s'agit-il pas d'une nouvelle «mode », qui, par le

1. Pour plus de précisions sur les références citées en notes, se reporter à la liste finale, infra, p. 152-155. Ici, Hargreaves Heap, 1989, p. 71. 
biais d'une rhétorique novatrice cherche à compenser sa faible valeur cognitive?

Afin de répondre à ces questions, il faudra distinguer deux éléments : d'un côté, la manière dont l'historien est censé mobiliser des modèles empruntés à l'économie et, de l'autre, la pertinence de l'un ou de l'autre schéma. Le premier but de ce travail consiste précisément à interroger la portée épistémologique des démarches néo-institutionnalistes. Il faudra en expliciter les hypothèses, rendre compte de leur cohérence et, finalement, mettre en évidence leurs principales implications pour l'historiographie. L'opposition, gnoséologique, théorique et idéologique, entre la notion d'État, ou celle d'institution en général, et la notion de «marché » a caractérisé la plus grande partie des débats économiques depuis au moins deux siècles. Or les interprétations néo-institutionnalistes et les théories des jeux vident cette opposition de sa raison d'être, dans la mesure où les institutions peuvent inclure aussi les règles du jeu, donc à la fois les rapports marchands et les contrats. Mais une fois acceptée cette démarche, quel rapport peut-on établir entre les systèmes économiques et leurs formes d'organisation?

Répondre à cette question signifie essayer de comprendre non seulement ce que ces approches nouvelles entendent par «institution », « marché », «travail », mais également la manière dont elles rapportent les «structures » aux «événements ». L'analyse néo-institutionnaliste traite certes des structures, mais pas des systèmes : elle mise sur les continuités. Au contraire, la théorie des jeux, avec ses choix réitérés et ses bifurcations, permet de prendre en compte les événements. Que se passe-t-il quand ces deux démarches se superposent?

L'enjeu de cette question est de taille : il consiste à expliciter le rapport entre la dynamique macroéconomique et macrohistorique, d'une part, et les comportements micro, d'autre part. Par exemple, une fois admis que des institutions hors marché et de multiples formes d'échange sont toutes également possibles et qu'elles sont loin d'être en opposition les unes avec les autres, peut-on encore affirmer que le capitalisme correspond à l'émergence d'une rationalité autre (comme l'affirmait l'économie classique jusqu'aux études de Karl Polanyi), ou bien s'agit-il de formes d'organisation et de stratégies différentes, mais dans le cadre d'une même rationalité ?

La notion d'information économique est ici cruciale, car elle présuppose une discussion préalable sur le rapport existant entre information, connaissance, traditions et conventions. Ces distinctions servent à comprendre la manière dont cette nouvelle historiographie envisage les ruptures et les continuités, et donc le temps historique. S'agit-il d'une véritable chronologie ou bien d'un temps logique? Et quel rapport peut-on établir entre la temporalité propre aux phases différentes d'un jeu et la temporalité historique? 
La première partie de ce travail étudie la manière dont les institutions ont été traitées par les principaux courants de l'histoire de l'économie. Après un bref rappel des thèses néo-classiques, sont analysées les approches néoinstitutionnalistes, en distinguant les études qui envisagent les institutions comme règles du jeu (propriété privée, propriété foncière, brevets, commerce, etc.) de celles qui s'intéressent aux entreprises et aux organisations. Ensuite, afin d'essayer d'évaluer la capacité des approches néo-institutionnalistes à rendre compte de la transformation des formes d'organisation et des systèmes économiques, sont examinés les marchés du crédit et du travail. Ce dernier aspect permet de discuter des différences entre conventions, institutions et marchés.

La seconde partie de ce travail prend en considération la théorie des jeux et ses applications à l'analyse historique, notamment à celle du commerce international. Une fois rappelés les travaux principaux sur ce sujet, leurs hypothèses, leurs catégories et leur cohérence sont ensuite discutées. Enfin, après avoir introduit des approches fondées sur la rationalité limitée, on tentera de mettre en question la notion d'information économique. À cette fin, il sera tenu compte à la fois des notions de «choix » et de «stratégie » propres aux jeux et des conceptions temporelles plus proprement historiques.

INSTITUTIONS ET COMPORTEMENT ÉCONOMIQUE

DE L'APPROCHE NÉO-CLASSIQUE AUX THÉORIES NÉO-INSTITUTIONNALISTES

\section{L'approche néo-classique}

Dans l'approche néo-classique traditionnelle, les institutions renvoient à l'aspect légal qui, lui, est opposé à l'aspect économique proprement dit, ce dernier étant identifié avec le «marché ». À partir des analyses d'Alfred Marshall et d'Arthur C. Pigou, les institutions publiques (l'État en particulier) sont censées n'intervenir que dans le cas d'imperfections du marché. En tout cas, le système économique ne peut aboutir à une solution optimale, correspondante à celle d'une économie parfaitement concurrentielle. Comme les institutions publiques, la concentration industrielle est aussi synonyme d'inefficacité. Les institutions sont donc envisagées comme des variables exogènes et les rapports entre les sujets privilégient les relations «horizontales» de marché, tandis que les liens verticaux tendent à être négligés. 
L'approche néo-classique, du moins dans sa version traditionnelle, ne se pose donc guère le problème de l'information économique. Une connaissance parfaite de l'état et de l'évolution des marchés est présupposée de la part du sujet économique. Les prix du marché offrent les informations nécessaires. Il n'y a pas d'incertitude; c'est pourquoi la monnaie agit simplement comme intermédiaire des échanges et non comme un bien en soi. Plus récemment, on a essayé de tenir compte de l'incertitude en introduisant la notion d'anticipations rationnelles (rational expectations). Si un sujet est rationnel, il cherche à acquérir le maximum d'informations, de manière à adopter la solution la plus efficace. L'information économique est en principe placée au cœur de cette approche mais, en réalité, elle constitue une variable non économique. Les sujets sont censés accumuler le maximum d'informations possibles et cela sans tenir compte des coûts d'acquisition et de gestion de l'information ni de son utilité. De ce fait, et malgré les prétentions affichées d'aboutir à une analyse dynamique, les modèles élaborés pour rendre compte de ces anticipations demeurent statiques : les erreurs commises dans les prévisions, selon Robert Lucas et les autres théoriciens des anticipations rationnelles, modifient les prévisions, mais non la manière dont elles se forment ${ }^{2}$. Dans ces conditions, les institutions sont traitées de la même manière que dans la pensée néo-classique traditionnelle : elles sont identifiées à l'État et comme telles sont opposées au marché. Les politiques économiques ne peuvent que s'adapter aux comportements du marché; autrement, elles provoquent une forte instabilité, et cela d'autant plus que les sujets peuvent anticiper le comportement de l'État. Malgré cette simplicité à la limite de l'irréalisme, la théorie néoclassique a longtemps été utilisée par les économistes et par les historiens; dans sa version marshallienne (les imperfections du marché), cette théorie a même ouvert les portes aux thèses néo-institutionnalistes.

\section{L’approche néo-institutionnaliste}

a) Les institutions comme règles du jeu.

Il est possible de distinguer deux principales tendances au sein de l'approche néo-institutionnaliste : d'une part, ceux qui - comme Douglass North et Dan Fogel — identifient les institutions hors marché avec les lois, les règles et la réglementation; d'autre part, ceux qui - comme Oliver Williamson - mettent l'accent sur les institutions privées, les entreprises notamment. Ces deux courants s'inspirent du travail de Ronald Coase sur les coûts de transaction. Selon cette approche, les institutions naissent jus-

2. Hargreaves Heap, p. 57-59. 
tement en réponse à ces coûts, qu'elles permettent de baisser sans aboutir pour autant à une solution optimale que seul un marché parfaitement concurrentiel peut assurer. D'une certaine manière, il est possible d'affirmer que les coûts de transaction et les organisations sont plus importants si le marché concurrentiel est moins développé ${ }^{3}$.

Comme l'a synthétisé Harold Demsetz ${ }^{4}$, les droits de propriété permettent d'internaliser les externalités. Ce processus a lieu quand les gains de l'internalisation dépassent les frais d'internalisation. À quel moment cette condition est-elle satisfaite? Le débat sur les formes de possession foncière permet de répondre à cette question et de rendre en même temps compte des différends existant, d'une part, entre les néo-classiques et les néo-institutionnalistes et, d'autre part, entre les différents courants néoinstitutionnalistes.

Donald McCloskey ${ }^{5}$ a observé que les champs ouverts et démembrés exprimaient l'aversion au risque des producteurs tout en leur assurant une protection contre les mauvaises récoltes. Stefano Fenoaltea a critiqué cette thèse en affirmant que la protection contre la mauvaise récolte était assurée par les réserves en blé, tandis que la fragmentation des lopins servait à minimiser les coûts de transaction. L'efficacité économique, et non une prétendue économie morale ni une aversion au risque, serait donc à l'origine du succès des champs ouverts à l'époque préindustrielle. Bien sûr, des changements institutionnels importants auraient permis, à partir du $\mathrm{XVII}^{\mathrm{e}}$ siècle, de baisser les coûts de transaction en rendant plus avantageux le remembrement et la privatisation des lopins ${ }^{6}$. Donald McCloskey et John Nash ont, à leur tour, répliqué que le stockage du blé était excessivement coûteux à l'époque préindustrielle; de ce fait, et compte tenu aussi du taux d'intérêt élevé, le démembrement des lopins était convenable du point de vue de la distribution du revenu, tandis qu'il pénalisait la productivité agricole et augmentait les coûts de transaction ${ }^{7}$.

Plus récemment, certains ont critiqué, d'une manière plus radicale encore, les thèses de McCloskey, en observant qu'elles présupposent que les agriculteurs médiévaux aient cherché à maximiser le profit et donc aient réagi négativement à une hausse du taux d'intérêt. Cette approche a donc été qualifiée d'anachronique ${ }^{8}$.

3. North, 1981. Voir aussi North et Weingast, 1989. Dans ce même courant, Rosenthal, 1992, montre comment, en France, les projets d'irrigation et de drainage ne furent pas concrétisés pendant un certain temps parce que les droits de propriété n'étaient pas suffisamment protégés.

4. Demsetz, 1988, p. 104.

5. McCloskey, 1976.

6. Fenoaltea, 1976 et Fenoaltea, 1988, repr. in Gustafsson, 1991, p. 107-172.

7. MCCloskey et Nash, 1984.

8. Komlos et Landes, 1991. 
Finalement, McCloskey a démenti avoir présenté le paysan médiéval comme un broker à la bourse de Londres; au contraire, a-t-il affirmé, précisément parce que les marchés des capitaux étaient peu développés, les paysans étaient obligés d'assumer d'autres formes d'assurance contre le risque de mauvaise récolte, à savoir le démembrement des lopins ${ }^{9}$.

Les différences entre les thèses néo-institutionnalistes et celles des néoclassiques traditionnels peuvent se résumer ainsi : les premiers généralisent les imperfections du marché et avec elles la définition de l' «institution ». Celle-ci ne se réfère plus seulement à l'État mais inclut toute sorte d'organisation et de règles, y compris les institutions privées et les contrats. De ce fait, les institutions n'entravent pas forcément les marchés et l'efficacité économique, comme l'affirment les néo-classiques, mais au contraire, permettent de les renforcer dans les cas où les « frictions » dues au fonctionnement du système sont importantes. Ainsi, Fogel montre que les politiques de stockage du blé adoptées par les autorités anglaises au Xvi siècle auraient été nécessaires, afin de distinguer l'irrationalité de l'imperfection du marché ${ }^{10}$.

La réplique néo-classique a consisté à montrer que les mesures de stockage et de vente des producteurs étaient rationnelles, car elles répondaient à l'objectif de maximiser le profit, et que la stabilisation des prix du blé dérivait moins de l'action du gouvernement que de l'intégration progressive des marchés européens ${ }^{11}$.

Autrement dit, si l'on suit l'approche néo-classique, le marché et sa rationalité peuvent se développer dans n'importe quel contexte institutionnel; l'État, les corporations et, de manière générale, des politiques arbitraires entravent les marchés et donnent vie à des solutions non optimales. Les institutions ne peuvent jouer un rôle efficace qu'en présence des imperfections du marché. Au contraire, les néo-institutionnalistes estiment que l'économie de marché constitue un cas particulier d'organisation qui peut s'imposer à d'autres formes d'échange, mais seulement là où les échanges impersonnels du marché présentent des coûts de transaction inférieurs à toute autre forme d'organisation qui internalise l'échange et l'information. Dans tous les autres cas, les associations entre producteurs, les corporations, les entreprises, tout comme les lois et les règles régissant l'économie permettent de réduire les coûts de transaction et d'améliorer l'efficacité du système dans son ensemble. La protection de la propriété privée en Angleterre aurait permis, par exemple, le développement des marchés et du capitalisme dans ce pays ${ }^{12}$.

\footnotetext{
9. McCloskey, 1991.

10. Fogel, 1992.

11. NIELSEN, 1997.

12. North, 1981 ; North et Thomas, 1973.
} 
Cette approche a été ensuite généralisée : non seulement le capital monétaire et le capital physique, mais même le «capital humain » exige une protection juridique adéquate. Les brevets permettraient d'optimiser les progrès techniques et leurs rythmes. Les brevets ont permis aux États-Unis d'atteindre un taux de développement particulièrement élevé à partir de la seconde moitié du XIX ${ }^{\mathrm{e}}$ siècle ${ }^{13}$. Cette conclusion présuppose, bien sûr, que le coût de production de la connaissance soit élevé, tandis que celui de la diffusion de la connaissance demeure limité. La notion d' « invention » présuppose donc deux concepts différents de l'information économique :

$1^{\circ}$ l'information comme découverte, c'est-à-dire la connaissance de $l^{\prime}$ « état de nature » du système;

$2^{\circ} 1^{\prime}$ information comme connaissance anticipée des réactions des autres.

Plus coûteux est l'accès à l'information et plus l'organisation de l'économie sera éloignée du marché concurrentiel. Or, comme c'était le cas pour les enclosures, cette littérature sur les brevets estime que la tutelle de la propriété privée est indispensable à la croissance économique, tandis que le rôle de l'entrepreneur capitaliste demeure à l'arrière-plan. La critique immédiate que l'on peut apporter à ce modèle consiste à mettre en doute le fait que l'institutionnalisation de la propriété privée fût indispensable au développement économique ; les innovations et l'esprit d'entreprise auraient, en effet, joué un rôle plus important. Avant d'examiner plus en détail ces critiques, il faut tout de même nuancer : car l'attention portée à la propriété privée n'est pas une caractéristique essentielle des thèses néo-institutionnalistes. Plusieurs auteurs, Bo Gustafsson ou Stefano Fenoaltea parmi d'autres, ont au contraire suivi cette approche pour démontrer le caractère non nécessaire de la propriété privée au développement économique. De ce fait, le véritable problème soulevé par cette approche n'est pas tant de savoir si la propriété privée a été réellement nécessaire à l'essor de l'économie de marché, mais plutôt si, une fois admis que les institutions ne sont pas forcément en opposition au marché, ce dernier se modifie avec l'affirmation du capitalisme? Autrement formulé, l'entreprise et son organisation sont-elles l'effet ou la cause de l'affirmation d'une économie de marché?

b) L'entreprise comme institution.

Le travail de Williamson ${ }^{14}$ constitue à la fois la référence et la généralisation de la plupart des études néo-institutionnalistes. La thèse principale de l'auteur peut se résumer ainsi : les institutions économiques du capitalisme, les entreprises en particulier, ont pour but et pour effet principal de

13. Sokoloff, 1988; Khan, 1995.

14. Williamson, 1985. 
réduire les coûts de transaction. Cette conclusion permet de remettre en cause toute une tradition de pensée, néo-classique aussi bien que marxiste, selon laquelle la concentration industrielle et les inégalités parmi les organisations s'expliquent par le désir des entreprises dominantes de jouir d'une rente ou d'une quasi-rente. L'équilibre ainsi obtenu serait alors plus coûteux et moins efficace par rapport à l'équilibre concurrentiel. Au contraire, selon Williamson, la concentration vise justement l'efficacité, car elle permet de réduire les coûts de transaction.

De très nombreuses «applications historiques » ont accompagné cette démarche. Ainsi, Bernard Elbaum et Walter Lazonick ${ }^{15}$ ont affirmé que l'avance prise à la fin du $\mathrm{XIX}^{\mathrm{e}}$ siècle par les États-Unis et l'Allemagne sur l'Angleterre s'explique par la concentration et l'intégration industrielles qui auraient permis de réduire les coûts de transaction liés à la rigidité grandissante des marchés. En Angleterre, ce processus n'aurait pas eu lieu, non pas à cause du conservatisme des entrepreneurs, mais du fait des contraintes institutionnelles.

Ce courant de pensée associe donc la naissance de l'industrie capitaliste au désir de baisser les coûts de transaction et notamment les frais de contrôle du travail. De ce fait, ces auteurs prennent leurs distances tant des thèses marxistes que de celles des néo-classiques qui associaient l'intégration capitaliste aux progrès techniques. Dans l'approche néo-classique, ces derniers constituent le facteur exogène principal qui permet d'expliquer la révolution industrielle (David Landes constitue le meilleur exemple de cette démarche). Au contraire, selon les néo-institutionnalistes, les progrès techniques constituent une conséquence des changements institutionnels : protection des droits de propriété, institution du parlement anglais, etc.

Ce débat sur la nature de la concentration des entreprises permet de mieux saisir les analogies et les différences entre l'approche néo-classique et celle des néo-institutionnalistes. La différence est apparemment facile à comprendre : selon les néo-classiques, la concentration vise à minimiser les coûts de production et à acquérir une position de rente ou de quasi-rente. Le résultat en est un équilibre peu efficace. Au contraire, les néo-institutionnalistes estiment que la concentration permet de réduire les coûts de transaction et que, de ce fait, elle est avantageuse pour l'ensemble du système économique. Cette dernière approche a le mérite de casser l'opposition traditionnelle entre marché et institutions (ces dernières étant le plus souvent identifiées avec l'État, ou avec les monopoles). Les sujets économiques privés par excellence, les entreprises, sont elles-mêmes des institutions. Le marché parfaitement concurrentiel est alors envisagé comme une hypothèse théorique qui ne trouve aucune confirmation dans la réalité. De

15. Elbaum et Lazonick, 1986. 
ce fait, les comportements non concurrentiels ne sont pas forcément contraires au principe d'efficacité; au contraire, dans la quasi-totalité des cas, les oligopoles et la concentration industrielle, ou bien les associations de commerçants, mais aussi la fragmentation des lopins répondent à un souci d'efficacité économique. Finalement, le marché n'étant plus anonyme, les rapports personnels assument une importance tout à fait déterminante.

Cependant, si le marché est une institution et si le sujet économique essaye toujours de minimiser ses coûts de transaction, comment peut-on distinguer le(s) capitalisme(s) des autres systèmes économiques?

Maxime Berg et Walter Lazonick montrent que le capitalisme peut prendre des formes organisationnelles différentes dans des contextes institutionnels également différents : concurrence en Angleterre; grande entreprise aux États-Unis; collectivisme au Japon. De même, la proto-industrie et l'industrie familiale présentent une variété de formes organisationnelles selon le contexte ${ }^{16}$. Cette approche remet en cause les classifications des systèmes économiques proposées par les littératures néo-classique, institutionnaliste et marxiste traditionnelles (capitalisme, économie paysanne, féodalisme, etc.). À leur place, nous trouvons plutôt une typologie des organisations, dont l'évolution est strictement liée à celle du contexte institutionnel. En réalité, l'approche néo-institutionnaliste a le mérite de briser la notion de «contexte historique », que les historiens avaient en bonne partie empruntée au ceteris paribus des économistes ou, dans les cas les plus «souples», aux approches positivistes. Dans tous ces cas, le « contexte » jouait dans l'explication historique un rôle à première vue rhétorique, mais en réalité crucial dans la détermination des liens de causalité. Les néo-institutionnalistes ont cassé cette boîte noire en faisant du « contexte» le protagoniste véritable de leurs analyses.

Malgré ces progrès, cette approche ne parvient pas à expliquer le rapport existant entre les changements institutionnels et les formes d'organisation du marché : par exemple, l'oligopole répond-il à une structure particulière des coûts de transaction ou bien à des hiérarchies socio-économiques préexistantes? En d'autres termes, les institutions sont-elles le résultat ou bien la source du comportement économique?

Comme toute démarche comparative, le néo-institutionnalisme exige une grille de lecture préétablie, qui serait censée être applicable à toutes sortes d'économies, du capitalisme japonais aux organisations commerciales médiévales. Cette grille s'appuie sur la notion clé de coûts de transaction. Le sujet n'agit plus dans une situation de connaissance parfaite, mais, au contraire, de rationalité limitée, à l'intérieur de laquelle il essaye de mini-

16. Lazonick, 1991, p. 253-301; Lazonick, 1990. Sur ce débat, voir Berg, 1991. 
miser les coûts de transaction. Étant donné cet objectif, il peut y avoir des cas de figure différents de combinaisons de marchés et d'institutions ${ }^{17}$.

Il reste à savoir si cette approche permet de rendre effectivement compte du passage d'une forme d'organisation à une autre sans tomber dans une explication purement tautologique qui justifierait toute solution historique sur la base des coûts de transaction. Il suffirait en effet qu'une institution (une loi, une forme d'organisation, une association) ait existé à un moment donné dans un contexte donné pour affirmer qu'elle répondait au souci de réduire les coûts de transaction. En est-il réellement ainsi? Afin de vérifier ce point, il est nécessaire de mieux comprendre le rôle que l'information économique joue dans les argumentations des néo-institutionnalistes : de quelle manière les sujets peuvent-ils acquérir les informations nécessaires? Quel rapport peut-on établir entre la circulation de l'information interne à l'organisation et les signaux provenant du marché?

Il est intéressant de commencer par envisager la manière dont la littérature néo-institutionnaliste a traité l'organisation du crédit. Ce sujet permet, en effet, de comprendre le rapport théorique entre variables monétaires et variables réelles, c'est-à-dire la nature, l'origine et l'emploi de l'information économique dans des contextes historiques différents.

\section{LE CRÉDIT ENTRE MARCHÉ ET INSTITUTIONS}

Les néo-classiques et les néo-institutionnalistes n'ont pas la même approche de l'information économique : pour les uns, elle n'a pas de véritable importance, tandis que, pour les autres, elle est fondamentale dans l'effort de réduction des coûts de transaction. Nous savons aussi que les anticipations sont, pour les néo-classiques, relativement statiques dans la mesure où un sujet rationnel qui utilise toutes les informations disponibles anticipe parfaitement les réactions des marchés. Les comportements réels coïncident avec ceux qui sont décrits par la théorie économique. C'est pourquoi, dans l'analyse de McCloskey, le risque constitue effectivement le paramètre principal à prendre en considération, tandis que Fenoaltea met l'accent sur l'incertitude. La notion de risque présuppose bien une distribu-

17. Cette démarche a deux conséquences majeures sur le plan de la théorie économique. En premier lieu, les équilibres à la Arrow-Debreu ne sont plus valables et l'architecture de l'équilibre général est bousculée : ni les décisions individuelles ni les prix de marché ne permettent à eux seuls d'aboutir à un équilibre général. En outre, la notion d'efficacité à la Pareto est remplacée par une autre : le résultat de l'activité économique peut être dit efficace si les sujets concernés peuvent effectivement négocier, réaliser et renforcer leurs décisions. Voir MiLGROM et Roberts, 1992, p. 21-24. 
tion de probabilité d'événements tous envisagés à l'avance par le sujet économique, tandis que l'incertitude tient compte des évolutions éventuelles de l'environnement économique et du comportement des autres sujets ${ }^{18}$. Cette distinction est d'une très grande importance pour les historiens. C'est manifeste dans le cas de l'analyse du marché du crédit.

En discutant du crédit rural en France au XVIII siècle, Jean-Laurent Rosenthal ${ }^{19}$ montre que les coûts de transaction élevés et la circulation difficile de l'information assignent aux marchands et aux notaires un rôle privilégié dans l'intermédiation financière. Au contraire, l'intervention de l'État sur les marchés des capitaux constituerait une source d'instabilité et finalement d'obstacle à la naissance d'un marché national des capitaux. Il y aurait, en ce cas, une distinction importante entre institutions privées et institutions publiques. Dans un marché non concurrentiel, les premières permettent d'améliorer l'efficacité du système qui, au contraire, serait réduite par les secondes.

Gilles Postel-Vinay a remarqué que cette approche, en faisant des institutions une «boîte noire », finit par expliquer le changement historique par en haut, c'est-à-dire par les réformes institutionnelles. Il s'ensuit une simplification excessive de la chronologie qui oppose un «avant» et un « après », la ligne de démarcation étant représentée par l'institutionnalisation de la propriété privée. Au contraire, si l'on suit la démarche de PostelVinay, les relations monétaires, le crédit et les marchés se seraient développés avant la Révolution française, celle-ci ayant fragmenté plutôt qu'unifié les marchés. À la place d'un avant et d'un après, on aurait des transformations de longue durée, avec des temps multiples et des circuits hiérarchisés du crédit et du travail ${ }^{20}$.

En réalité, l'analyse de Rosenthal présuppose une situation dans laquelle les sujets économiques essayent d'atteindre leur but (minimiser les coûts de transaction) dans une situation suboptimale. L'information économique sert à expliquer comment un équilibre est atteint (en ce cas, la mise en place du système du crédit prérévolutionnaire). Cependant, la manière dont les sujets traitent les informations et leur attitude optimisante est anhistorique. L'auteur affirme que les institutions jouent un rôle crucial dans l'équilibre et dans l'évolution du système économique. Toutefois, une institution par excellence, la monnaie, n'a pour ainsi dire pas de place dans la détermination de l'équilibre. On parle d'économie monétaire, mais finalement on demeure dans un système de troc déguisé. C'est pourquoi le rôle des institutions demeure ambigu. D'une part, elles sont présentées comme des variables exogènes: l'institution et les changements institutionnels

18. Sur cette différence, voir KNIGHT, 1964.

19. Rosenthal, 1994.

20. Postel-Vinay, 1997. 
affectent le comportement économique des sujets. Mais, d'autre part, les institutions se modifient selon les intérêts et les préférences des sujets. Cette impasse théorique rend incertaine l'explication du changement : on ne comprend pas bien si, par exemple, l'affirmation du capitalisme est dû au triomphe des règles du marché sur des institutions peu efficaces ou si, au contraire, les institutions auraient permis l'affirmation des rapports de marché. Afin de répondre, il faudra se placer à la frontière entre la notion de marché et celle d'institution. L'analyse des conventions sur le marché du travail peut ici se révéler féconde.

LES CONVENTIONS SUR LE MARCHÉ DU TRAVAIL

Vers le milieu des années 1960 déjà, George Stigler ${ }^{21}$ montrait que la présence de salaires différents pour des travaux égaux reflétait les coûts de circulation et donc d'acquisition de l'information de la part des travailleurs et des employeurs. Si le marché a une extension spatiale plus importante, il faudra aussi tenir compte des frais de transport de l'information (orale, écrite, etc.). Cette approche permet de rendre compte des différences salariales dans des contextes différents; cependant, elle présuppose que les travailleurs, comme les entrepreneurs, adoptent un comportement qui cherche à optimiser la recherche, puis l'emploi des informations requises, c'est-àdire à minimiser les coûts de transaction.

Un résultat semblable a été obtenu en suivant une perspective différente, axée plutôt sur les problèmes de surveillance et de négociation des contrats. Paul Milgrom et John Roberts considèrent les contrats implicites comme une forme particulière de contrats incomplets, qui posent donc des problèmes de hasard moral. Ces difficultés peuvent se résoudre soit par des contraintes (sanctionner le sujet qui ne respecte pas son engagement) soit par des incitations (offrir une rémunération plus élevée). Les contrats implicites naissent en réponse aux anticipations que chacune des parties se forme en situation d'information incomplète et de rationalité limitée. Les contrats implicites peuvent être envisagés comme un instrument permettant d'économiser les coûts de négociation et de rationalité limitée. Par exemple, ce sont justement ces règles non écrites qui permettent de rendre compte, selon les auteurs, de la persistance de la culture corporative.

Les historiens ont eu recours à ce concept de «contrat implicite » qui a été envisagé comme une forme d'assurance, soit contre les oscillations de

21. Stigler, 1968 et 1982. 
la demande de travail, soit contre celles de l'offre et des prix des biens d'alimentation. Deux positions principales se sont alors dégagées : certains estiment que les contrats implicites concernent les services offerts par les employeurs ou bien par l'État; d'autres mettent au contraire l'accent sur les formes d'assurance entre les travailleurs, telles que les prêts entre familles, la charité, etc. ${ }^{22}$. Nous retrouvons là des orientations tout à fait semblables à celles que nous avons discutées auparavant sur le système d'approvisionnement anglais au $\mathrm{XVII}^{\mathrm{e}}$ siècle. Dans le premier cas, les institutions publiques complètent le marché; dans le second cas, ces institutions auraient été inefficaces et les travailleurs auraient développé leurs propres formes d'assurance.

Cette littérature sur le marché du travail permet de mieux comprendre la différence entre la notion d'institution et celle de convention dans les travaux récents d'histoire de l'économie. Certes, les institutions peuvent être envisagées aussi bien comme formes d'organisation que comme ensemble de conventions. Dans le cas du marché du travail, ces deux aspects sont souvent superposés : que l'on songe aux corporations, à l'État, aux coopératives, et même aux familles et aux entreprises. Ces organisations s'appuient nécessairement sur des conventions et des règles qui ne sont pas nécessairement écrites, ni formulées de manière explicite par les contractants. L'inverse n'est pourtant pas toujours vérifié : les normes ne donnent pas forcément vie à des organisations. Quel rapport peut-on alors établir entre les unes et les autres?

Les conventions sont, en général, évoquées comme formes de coordination supplétive aux interactions marchandes. Cependant, à l'intérieur de cette démarche commune, au moins deux approches principales peuvent se dégager. Selon une certaine littérature anglo-saxonne ${ }^{23}$, la présence des conventions, et donc l'impasse dans la coordination par le marché, n'infléchit pas l'individualisme méthodologique, ni le recours à une rationalité instrumentale. Au contraire, ces éléments sont remis en question dans les travaux de plusieurs «conventionnalistes » français ${ }^{24}$. Ici, étant donné l'objet d'investigation (l'économie néo-institutionnaliste), l'attention sera portée essentiellement sur les travaux anglo-saxons.

Plusieurs auteurs ont évoqué les contrats implicites afin de rendre compte des contrats de formation, dont la durée et les conditions sont expliquées par les informations à la disposition de l'employeur plutôt que par la productivité du travailleur ${ }^{25}$. On part du constat que sur le marché du tra-

22. KIESLING, 1996; Boyer, 1990.

23. Schelling, 1977.

24. Voir le numéro spécial de la Revue économique, 1989. Pour une comparaison avec l'approche anglo-saxonne, voir Gomez, 1994.

25. Hamilton, 1996. 
vail les rapports sont caractérisés par des asymétries informationnelles, auxquelles les employeurs essayent de faire face par des contrats de formation. Un employeur tendrait à prolonger la durée d'un contrat et à y inclure la formation des garçons les plus jeunes, en fonction de leur productivité future, plutôt que passée, ainsi qu'en fonction de l'information et du contrôle direct qu'il peut exercer sur les jeunes employés. Autrement dit, le contrat de formation permet de réduire les risques de hasard moral.

D'autre part, cette exigence des employeurs tend à rencontrer celle des travailleurs : plus jeunes ils sont et plus durables sont les emplois qu'ils cherchent. Ces exigences convergentes du côté de la demande et de l'offre de travail, tout comme la création et la circulation de l'information économique au sujet des jeunes travailleurs parmi les employeurs, permettent d'expliquer le peu de protestations légales de la part de ces derniers à l'égard du non-respect des engagements pris par les travailleurs.

Des résultats tout à fait analogues sont obtenus dans l'étude comparative de Bernard Elbaum sur les contrats de formation en Angleterre et aux États-Unis ${ }^{26}$. Selon lui, la période de formation initiale persiste au cours du $\mathrm{XIX}^{\mathrm{e}}$ siècle en Angleterre, et non aux États-Unis, à cause de la plus forte tradition corporative anglaise. Ce résultat présuppose une structure différente des coûts de transaction. Afin de franchir les obstacles législatifs et de baisser les coûts de transaction, les entreprises anglaises ont recours à des contrats oraux. Cette solution pose bien sûr un problème de contrôle et de respect des engagements pris. Les entreprises investissent en capital humain et font circuler les informations concernant les employés afin de mieux les contrôler. Les travailleurs à leur tour peuvent se former et résoudre en même temps les problèmes d'information asymétrique. Ils auront alors tout intérêt à respecter les engagements pris.

Autrement dit, la coopération parmi les employeurs ou les marchands, notamment par le biais de la circulation de l'information économique, permet de résoudre les problèmes de hasard moral et en général d'agence. Une implication théorique est que l'information, comme tout autre bien, peut être accumulée et ce capital d'informations permet de réduire les coûts de transaction en donnant à son possesseur des avantages comparatifs par rapport à ses concurrents ${ }^{27}$.

Une conséquence pour la théorie du développement et pour l'histoire de l'économie est que, à la différence de l'affirmation d'Adam Smith et des économistes «classiques», le capitalisme et l'industrialisation ne s'associent guère à la spécialisation et à la division du travail, mais plutôt à des changements dans l'organisation du travail qui auraient permis de réduire

26. Elbaum, 1989.

27. STIGLeR, 1968, p. 262. 
les coûts de transaction, et notamment les frais de surveillance des travailleurs. Ces changements organisationnels auraient à leur tour rendu possibles les progrès techniques et le processus de spécialisation du travail ${ }^{28}$.

La naissance du capitalisme ne s'accompagne pas de celle d'une rationalité autre, mais elle témoigne de l'émergence de stratégies nouvelles, conformes au contexte institutionnel nouveau. Une différence majeure s'y manifeste par rapport à « la grande transformation » de Polanyi qui mettait l'accent sur l'existence de formes historiques différentes des marchés et de la rationalité économique. La minimisation des coûts de production constituait à la fois l'expression, la source et la conséquence principale de la grande transformation. Le marché capitaliste exprimait ainsi un phénomène structurel imprégnant les composantes différentes de la vie économique, sociale et culturelle ${ }^{29}$. Les approches néo-institutionnalistes tiennent par contre à distinguer nettement les aspects économiques des croyances et des convictions telles qu'elles ont été étudiées par les anthropologues et les sociologues. Le sujet qu'elles décrivent vise toujours à minimiser les coûts de transaction. Il n'y a pas de « grande transformation » ni de ruptures historiques fortes. Le capitalisme se distingue des autres formes historiques moins pas le comportement économique des sujets que par les règles du jeu et par ses formes organisationnelles et institutionnelles. La force de cette approche par rapport à celle de Polanyi est qu'elle brise une chronologie simpliste fondée sur un avant et un après et surtout sur une généralisation du changement (la «grande transformation») qui ne trouve finalement guère de correspondance dans les transformations historiques réelles. Surtout, Polanyi ne prenait pas en considération le problème de l'information et de l'incertitude : de ce point de vue, sa notion du marché autorégulateur reproduisait la représentation néo-classique de la concurrence parfaite.

Cependant, par rapport à Polanyi, l'approche néo-institutionnaliste connaît aussi une limite majeure : elle réduit les rapports hiérarchiques et les problèmes d'agence à des problèmes de coordination parmi des sujets ou des groupes ayant des intérêts éventuellement divergents, mais de force égale. Le rôle du pouvoir dans la mise en place des hiérarchies socioéconomiques est délaissé. S'agit-il d'une incompatibilité épistémologique ou est-il possible d'améliorer les modèles néo-institutionnalistes de manière à tenir compte des rapports de pouvoir?

Gustafsson a récemment essayé de combler cette lacune. Selon cet auteur, il est possible de «traduire les catégories de Marx dans celles de l'économie institutionnaliste et néo-institutionnaliste ». Il observe que la théorie de Marx explique le changement historique par les progrès dans les

28. NORTh, 1981.

29. Polanyi, 1983. 
rapports de production et par la hausse de la productivité. Cependant, l'évolution historique ne confirme pas cette théorie. L'auteur suggère alors d'intégrer l'explication néo-institutionnaliste (le changement lié aux coûts de transaction) à l'analyse des rapports de pouvoir. Gustafsson explique ainsi l'existence, puis le déclin des guildes médiévales : ces organisations servent à garantir une certaine qualité du produit, indispensable pour assurer un revenu stable à l'artisan et un bien-être plus élevé à la communauté. Les guildes maximisent l'emploi et cherchent un revenu stable. Elles tendent donc à maximiser leur revenu moyen. Du fait des barrières à l'entrée sur le marché, d'autres producteurs, qui ne sont pas admis dans les guildes, développent d'autres formes de production, fondées sur le travail salarié et cherchent à maximiser le profit. Cette solution est rendue possible par la hausse de la demande et donc par la moindre importance de la qualité du produit. Selon Gustafsson, les guildes ne déclinent pas parce qu'elles sont irrationnelles, mais parce que leur forme de rationalité, à partir d'un certain moment, devient moins efficace que la rationalité capitaliste. L'entreprise capitaliste, avec la hausse de la demande, permet de mieux résoudre les problèmes de surveillance par rapport à l'exploitation artisanale $^{30}$.

Cette démarche semble certes combler une insuffisance de la théorie néo-institutionnaliste, à savoir l'absence des rapports de pouvoir dans les hiérarchies des organisations économiques. En même temps, l'explication $\mathrm{du}$ changement historique demeure une justification a posteriori. Toute forme d'organisation et d'institution peut trouver sa justification dans le fait que, dans un contexte donné, elle permet de minimiser les coûts de production et de transaction. Le problème est que ces coûts à eux seuls paraissent insuffisants pour rendre compte de l'évolution historique; cela d'autant plus que, dans l'approche néo-institutionnaliste, le rapport entre évolution économique et dynamique des institutions demeure ambigu, l'une et l'autre jouant tantôt comme variable indépendante, tantôt comme variable dépendante. Le discours devient ainsi circulaire : les rigidités du marché et des institutions sont à l'origine des organisations et des institutions; en ce cas, de quelle manière ces dernières peuvent-elles s'adapter aux exigences de l'économie?

L'impression est que la rigidité et la flexibilité prétendues de l'une ou de l'autre forme d'institution servent en réalité moins à expliquer la dynamique historique qu'à légitimer le rôle privilégié et quasiment unique assigné à une boîte noire, les coûts de transaction, dans l'explication historique. De quelle manière peut-on sortir de cette impasse?

Il est désormais avéré que les notions de contrats implicites et de contrats incomplets couvrent des cas de figures différents. Milgrom et

30. Gustafsson, 1991, p. 4, 38, 82 et 103. 
Roberts évoquent les facteurs présents lors de la négociation du contrat et qui empêchent les rapports économiques de se conformer uniformément aux prescriptions néo-classiques traditionnelles. La rationalité limitée est l'un de ces facteurs : on ne peut pas tout prévoir, car il y a des événements exceptionnels ainsi que des motivations non économiques des sujets. De plus, les sujets peuvent renier un contrat, surtout s'il est incomplet ou implicite. Enfin, il est possible que les parties contractantes ne fassent pas circuler les informations. En ce cas, les solutions proposées (coopération parmi les producteurs ou les marchands, contrats implicites, etc.) ne sont plus praticables. L'acquisition des informations devient alors cruciale ${ }^{31}$. Mais de quel type d'information s'agit-il? Et quel rapport peut-on établir entre l'accès à l'information, les coûts de transaction et les changements historiques?

Pour répondre, il vaut mieux comprendre la manière dont l'histoire économique esquissée par ces analyses traite l'événement et sa relation avec les perceptions des individus. Il faut aussi expliquer la manière dont les individus forment leurs perceptions et affichent leur objectifs.

\section{LA THÉORIE DES JEUX ET L'HISTOIRE DE L'ÉCONOMIE}

Récemment, une nouvelle approche de l'histoire de l'économie a commencé à s'imposer; elle accentue davantage le rôle de l'information économique dans l'explication historique, en ayant recours à la théorie des jeux. Dans l'analyse historique, ces instruments ont été utilisés surtout pour discuter du commerce international à l'époque préindustrielle. Au début des années 1970 déjà, Douglass North et Robert Thomas soulignaient que les corporations commerciales cherchaient à réduire les coûts de transaction. Plus récemment ${ }^{32}$, fut précisé le rôle que les grandes compagnies commerciales à l'époque moderne jouèrent dans la réduction des coûts de transaction dus à la recherche puis au contrôle des agents et des correspondants commerciaux.

Dans ce sillage, Avner Greif ${ }^{33}$ s'est demandé comment les marchands ont pu disposer d'agents de commerce fiables. Il propose à ce sujet une étude comparative entre les marchands génois, allemands et maghrébins. Relativement à ces derniers, il observe que dans le commerce international médiéval les marchands maghrébins arrivent à faire face aux problèmes

31. Milgrom et Roberts, 1992, p. 159-161.

32. Carlos et Nicholas, 1988.

33. GREIF, 1989, 1993 et 1998. 
d'agence en présence d'asymétrie de l'information en s'alliant entre eux plutôt qu'en exerçant des punitions légales à l'égard de leurs agents. Ces derniers n'ont d'ailleurs aucun intérêt à ne pas respecter leurs engagements pris parce que les commerçants maghrébins font circuler les informations sur les agents. De ce fait, le maintien de la réputation devient un élément essentiel dans les rapports économiques.

Greif en tire la conclusion que les alliances parmi les commerçants maghrébins n'avaient pas pour but l'acquisition d'une position monopoliste, mais l'efficacité, c'est-à-dire la réduction des coûts de transaction liés à la gestion des agents commerciaux. Nous retrouvons ici la même conclusion que celle des auteurs néo-institutionnalistes au sujet des compagnies commerciales à l'époque moderne. Le recours à la théorie des jeux serait-il donc un artifice purement rhétorique?

Comme pour tout autre modèle théorique « appliqué » à l'investigation historique, il faut distinguer ici les caractéristiques propres au schéma théorique de l'usage qui en est fait. Plusieurs auteurs ${ }^{34}$ se sont livrés à des comparaisons fondées sur l'un ou sur l'autre modèle de théorie des jeux. Dans ces exercices, cependant, le souci de garder la cohérence du modèle théorique s'est imposé sur celui de respecter les témoignages hétérogènes des sources et la complexité du cadre référentiel. Autrement dit, la théorie des jeux a été utilisée moins comme un schéma heuristique susceptible de proposer des interrogations nouvelles, que comme modèle cohérent auquel plier les sources. L'effort de généralisation a parfois conduit à une banalisation des résultats et à une lecture réductrice des sources.

Cela signifie-t-il que l'historien des faits économiques n'a rien à apprendre de la théorie des jeux considérée comme un schéma heuristique?

D'emblée, il est clair que, par rapport aux approches néo-classiques et néo-institutionnalistes la théorie des jeux n'envisage pas un agent (individuel, une entreprise, etc.) confronté au «marché », mais, au contraire, permet de rendre compte de l'interaction entre agents. C'est une voie de sortie possible hors de l'opposition traditionnelle entre micro et macro (histoire). En particulier, des phénomènes de réputation et d'intégration dans la communauté jouent un rôle important. Les institutions hors marché n'entravent pas forcément la mise en place d'une économie de marché et, de manière générale, les sentiers économiques peuvent se différencier même si le « contexte» est semblable. L'information économique joue un rôle crucial dans la mesure où la connaissance est imparfaite et des variables non économiques interviennent dans les choix plus spécifiquement économiques. Le même « milieu » peut donner vie à des perceptions différentes, selon le passé de l'agent, ses convictions et ses «croyances» (believes).

34. Greif, 1998; Greif, Milgrom et Weingast, 1994. 
Dans ce contexte, à la différence des anticipations rationnelles et des approches néo-institutionnalistes, où chaque sujet est pris individuellement et où davantage d'information ne peut qu'améliorer ses capacités décisionnelles, la théorie des jeux ne prend pas seulement en considération les coûts de production et l'utilité de l'information, mais elle montre que davantage d'information peut éventuellement porter préjudice au sujet qui l'acquiert ${ }^{35}$.

De quelle manière l'historien peut-il tirer bénéfice de ces suggestions? Toute réponse doit satisfaire à au moins deux exigences : expliciter les catégories sous-jacentes aux différents modèles de théorie des jeux; les inscrire dans une temporalité propre au discours historique.

Relativement au premier point, une question peut se poser : qu'en est-il de la rationalité économique dans la théorie des jeux? Apparemment, la rationalité consistant à maximiser le profit et/ou à minimiser les coûts de production est mise en cause. Mais, pour autant, d'autres formes de rationalité sont-elles acceptables?

D'autre part, nous avons montré que l'approche néo-institutionnaliste n'est pas capable de rendre compte de l'évolution du processus de connaissance du sujet économique : cette impasse serait-elle résolue par la théorie des jeux?

Cette question en implique une autre : la plupart des études historiques inspirées des modèles néo-classiques et néo-institutionnalistes arrivent difficilement à expliquer l'écroulement d'un système sans tomber dans une justification tautologique. Un traitement différent de l'information économique et du processus de connaissance du sujet permet-il de sortir de cette impasse ? Ce qui est en cause c'est la notion d' « histoire » sous-jacente à ces démarches et, en particulier, le rôle qu'elles assignent à l'événement par rapport aux «structures » et la conception du temps qu'elles utilisent (chronologique ou bien logique).

LES JEUX ET LA DYNAMIQUE HISTORIQUE

Les études citées ont recours à des modèles différents de théorie des jeux ; mais il s'agit toujours de jeux non coopératifs. Un jeu est dit coopératif si un mécanisme qui impose les accords est disponible ${ }^{36}$. John Nash a élaboré le premier une théorie des jeux non coopératifs ${ }^{37}$. Cependant, dans

35. Pour une démonstration, voir GibBons, 1992, p. 69-70.

36. Aumann, 1985.

37. NASH, 1951. 
son approche originale, cette théorie présuppose une information complète des joueurs et surtout que la rationalité, la structure du jeu et les convictions des joueurs sur le comportement des autres soient des connaissances communes à tous les joueurs ${ }^{38}$.

Par la suite, plusieurs auteurs ont essayé de développer des modèles qui affaiblissaient l'une ou l'autre de ces hypothèses. Par exemple, les «jeux répétés » imaginent qu'un jeu soit composé de plusieurs phases. Chaque joueur modifie alors son comportement à partir des réactions des autres joueurs; la solution dépend aussi de l'histoire du jeu (on parle, en ce cas, de dépendance temporelle stratégique). Le processus d'acquisition des informations devient alors crucial. Dans ce contexte, il faudra distinguer entre information complète et information parfaite ${ }^{39}$. Mais, en tout cas, la notion de stratégie devient plus complexe que dans les jeux statiques. Gibbons la définit ainsi : dans le cas d'un jeu répété, fini ou infini, la stratégie d'un joueur dicte son action à chaque étape du jeu et pour toute histoire possible du jeu jusqu'à l'avant-dernière étape du jeu.

Que se passe-t-il si l'information est incomplète? En ce cas, les fonctions de paiement des joueurs ne sont plus connaissance commune, c'est-àdire que les joueurs ne connaissent pas d'emblée les objectifs des autres joueurs. Cependant, il est possible de se faire une idée de ces objectifs en observant leur comportement (on parle ici d'approche bayésienne). Mais comment être sûr que ces observations soient fiables et que donc un équilibre puisse être atteint?

John Harsanyi a imaginé que, contrairement au modèle de Nash, le sujet agit en se disant : «Peu importe ce qui se passe; je fais de mon mieux et je suppose que chacun fait de même ${ }^{40}$. » Cette approche laisse cependant un problème ouvert : que veut dire «faire le mieux » ? Répondre à cette question signifie distinguer le rôle de l'information acquise de celui des convictions initiales des joueurs, lorsque ces derniers déterminent leurs stratégies $^{41}$. Ainsi, nous avons montré que les jeux à information complète, mais asymétrique, ont été largement utilisés dans l'analyse historique du commerce international. En ce cas, les jeux répétés infinis assurent un équilibre stable grâce au mécanisme de réputation de chaque agent. Si un joueur répète ses comportements, ou si ses réactions sont prévisibles, il acquiert

38. Définition de connaissance commune : un joueur sait qu'un autre sait, qu'un autre sait..., etc. La fonction de paiement est une fonction numérique sur l'ensemble de l'issue du jeu. Les issues sont déterminées par chacun des joueurs.

39. GibBons, 1992, définit les jeux à information complète comme ceux où les fonctions de paiement des joueurs sont une connaissance commune; par contre, la connaissance parfaite signifie que le joueur connaît l'histoire du jeu, c'est-à-dire, les coups précédents.

40. Harsanyi, 1967.

41. Kreps et Wilson, 1982. Pour un panorama des critiques et du débat issu de cette approche, voir FudenBerg et TiRole, 1991. 
une certaine réputation aux yeux des autres joueurs qui, par conséquent, modifient leurs stratégies. On a, en particulier, essayé de déterminer les conditions où aucun des sujets impliqués par un accord ou un contrat ne trouve convenable de tricher. Ces conditions sont que l'information circule et qu'il existe la possibilité d'avoir recours à d'autres partenaires ${ }^{42}$.

En réalité, l'hypothèse d'asymétrie informationnelle peut susciter des cas de figure différents : une fois l'accord fixé (information complète), un des joueurs peut cacher à l'autre une action, ou bien une information. On peut aussi imaginer qu'une information est incomplète : on parvient à un accord sans qu'un des joueurs connaisse les stratégies et les préférences de l'autre joueur. Les modèles de Greif se réfèrent à la première typologie : les informations sont complètes; le principal doit chercher à contrôler l'activité du subordonné; il faudra donc tenir compte des coûts de l'information, ainsi que des incitations et des punitions éventuelles pour faire face à la tricherie éventuelle du subordonné.

Dans son analyse des commerçants maghrébins, Greif utilise des jeux répétés infinis avec information complète et parfaite, mais avec surveillance imparfaite (existence d'asymétrie informationnelle), tandis que l'information est imparfaite à l'intérieur de la Hanse allemande qui n'arrive pas, de ce fait, à un équilibre auto-exécutoire. Or, les jeux répétés infinis avec information parfaite permettent de montrer que des formes de coopération efficaces sont possibles même là où elles seraient impossibles si le jeu n'était pas répété. Un exemple de ce dernier cas est justement offert par l'analyse de Avner Greif, Paul Milgrom et Barry Weingast sur les foires en Champagne : les agents et les marchands se rencontrent occasionnellement, à la foire; le mécanisme de réputation n'est alors pas suffisant pour assurer la stabilité de l'équilibre sans une forme de coercition extérieure, en l'occurrence une cour des marchands. Par contre, chez les commerçants maghrébins, les conditions d'un contrat social sont assurées par les formes des accords et il n'y a pas besoin d'une autorité extérieure (comme en Champagne) pour assurer l'équilibre et le respect des contrats.

Cependant, Greif, Milgrom et Weingast montrent aussi que, dans le cas où les informations circulent lentement, des accords multilatéraux peuvent offrir une solution stable mais qui n'est pas efficace, dans la mesure où le volume du commerce et les frais ne sont pas à leurs niveaux optimaux.

42. On parle de trigger strategy quand un joueur i est porté à coopérer jusqu'à ce qu'un autre joueur ne s'éloigne de la solution coopérative; en ce cas, la trigger strategy suggère de ne plus coopérer dans l'avenir. Si tous les joueurs adoptent cette stratégie, alors la solution du jeu sera coopérative, voir GibBons, 1992, p. 97. Cependant, Abreu, 1988, a montré que la manière la plus efficace pour empêcher un joueur de s'écarter d'une certaine stratégie consiste à le menacer de la punition la plus dure imaginable si son adversaire s'éloigne aussi de la stratégie indiquée. Cette solution permet d'obtenir des fonctions de paiement moyens plus élevés que ceux de la trigger strategy. 
Cette dernière condition est satisfaite si la ville, même si elle se trouve dans des conditions de pénurie extrême, fait respecter l'embargo. En ce cas, son comportement devient connaissance commune, le mécanisme de réputation se met en place et les anticipations de tous les agents sont compatibles entre elles ${ }^{43}$.

Ces travaux ont donc recours à des modèles particuliers de jeux : il n'y a pas d'incertitude concernant le passé et les informations se traduisent aussitôt en connaissance ${ }^{44}$. La rationalité demeure instrumentale et certaines limites que nous avions déjà indiquées pour la littérature néo-institutionnaliste demeurent: on n'arrive pas à distinguer entre les systèmes économiques et les formes d'organisation et, de ce fait, on n'envisage pas la possibilité d'une évolution des formes de rationalité économique. Autrement dit, ces travaux s'appuient sur une prémisse forte : que les agents économiques, dans n'importe quel lieu et n'importe quel temps, cherchent à maximiser leur efficience. Du coup, l'explication offerte du devenir historique est tautologique, puisqu'on cherche à démontrer précisément que l'efficience constitue le but des agents et de leurs associations. Finalement, on peut se demander si cette démarche est intrinsèque à la théorie des jeux.

Avant de répondre, il faudra examiner, d'une part, ces travaux sur l'information économique qui ont proposé des formes différentes de rationalité et, d'autre part, les tentatives pour distinguer l'information de la connaissance (analyse de l'apprentissage).

\section{RATIONALITÉ LIMITÉE ET CONNAISSANCE}

Comme il a été montré, les approches traditionnelles de la théorie des jeux délaissent le processus de connaissance. Certains modèles traitent ce processus comme un appendice de la maximisation de l'utilité en condition d'incertitude. D'autres imaginent des sujets ayant des critères de connaissances ad hoc ${ }^{45}$. Dans un cas comme dans l'autre, la rationalité économique demeure purement instrumentale. Est-il possible de suivre une autre approche?

43. Greif, Milgrom et Weingast, 1994. Plusieurs auteurs ont déjà mis en évidence le peu de réalisme de cette situation dans laquelle les joueurs aboutiraient à un équilibre à la valeur moindre pour chacun d'entre eux, voir Abreu, Milgrom et Pearce, 1991. Il est cependant possible d'indiquer les conditions pour qu'un équilibre soit atteint, voir Fudenberg et Tirole, 1991 , p. 514 et 517.

44. Dans les jeux répétés, le passé influence l'équilibre final sans toucher cependant à l' « environnement physique ». Par contre, les équilibres de Markov tiennent compte de cette éventualité et, de ce fait, sont applicables dans des situations plus restreintes que celles des équilibres de Nash, voir FudenBerg et Tirole, 1991, p. 514.

45. KiEFER et NyARKo, 1995. 
À partir des années 1950, Herbert Simon a proposé sa notion de rationalité limitée (bounded rationality) ${ }^{46}$. Les perceptions du sujet deviennent centrales : même si une solution est considérée comme objectivement erronée, c'est-à-dire irrationnelle selon la théorie traditionnelle, elle peut être poursuivie en tant que solution bonne et juste ou, en tout cas, conforme aux postulats du sujet, qu'ils soient, par exemple, non capitalistes, voire non économiques. Simon distingue entre rationalité limitée substantielle et rationalité limitée procédurale. La rationalité est limitée substantiellement quand le sujet ne dispose pas de toutes les informations. Une fois ces dernières obtenues, il se comporte selon les prescriptions de la théorie économique standard. Cette forme de rationalité limitée suppose que l'information se traduise aussitôt en connaissance ${ }^{47}$.

La rationalité procédurale analyse, par contre, la manière dont les sujets traitent l'information ${ }^{48}$. Le sujet peut disposer d'informations, cependant il rencontre des limites dans leur traitement, par exemple du fait de catégories et donc de rationalités économiques différentes. Dans ce contexte, la prédécision, sur la base des expériences précédentes, sert à mieux allouer les efforts et le temps; le résultat ne sera pas forcément d'accorder la priorité au raisonnement et donc à la rationalité économique classique. Une meilleure information peut alors pousser le sujet à adopter un comportement qui n'est pas conforme aux résultats attendus par la théorie standard (néoclassique, néo-institutionnaliste et théorie des jeux traditionnelle).

Il est important de distinguer cette approche de celle des néo-institutionnalistes (d'autant plus que Williamson cite Simon comme un de ses principaux inspirateurs). Dans un souci de "réalisme», ces derniers admettent que la concurrence parfaite n'existe pas dans la réalité et que, de ce fait, il faut envisager des stratégies suboptimales. En réalité, Williamson utilise la rationalité limitée substantielle : elle exprime des connaissances imparfaites et des asymétries informationnelles qui limitent non l'intention rationnelle du sujet, mais la rationalité ex-post de son action. La rationalité reste strictement instrumentale (l'objectif consiste toujours à minimiser les coûts de transaction) et l'on exclut les aspects non économiques sur lesquels Simon avait mis davantage l'accent. Les aspects empruntés à l'anthropologie ou à la sociologie servent alors à confirmer plutôt qu'à nier la suprématie de l'économie et de sa rationalité. Tandis que les problèmes des « croyances » dans la théorie des jeux, tels que nous les avons évoqués plus haut, demeurent irrésolus, nous assistons à la tentative explicite de réappropriation de la part de l'économie de toute cette littérature qui, à partir des années 1960, avait essayé d'intégrer les postulats de l'économie

46. SimON, 1982.

47. SAlmon, 1995.

48. Selten, 1978, a été un des premiers à appliquer cette notion à la théorie des jeux. 
politique aux approches anthropologiques. Les traditions sont alors traduites en distribution de probabilités à leur tour exprimées sous forme de maximisation de l'utilité ou/et de minimisation des coûts. En ce cas, la rationalité est limitée parce que le sujet ne dispose pas d'une connaissance complète et parfaite qui permette d'adopter une rationalité optimisante.

Est-il possible de dépasser cette approche? Tenir compte d'autres formes de rationalité économique équivaut à distinguer l'information de la connaissance et à envisager ainsi la manière dont les agents économiques forment et modifient leurs perceptions. Ces dernières années, une riche littérature en matière d'apprentissage (learning) a été produite, le plus souvent dans le cadre de la théorie des jeux. Cette littérature mériterait à elle seule un ouvrage; parmi ses caractéristiques essentielles, seules seront rappelées ici celles qui sont le plus susceptibles d'intéresser notre sujet.

Déjà en 1989, on distinguait entre information et connaissance en remarquant que le secteur de « recherches et développement » d'une entreprise non seulement donne vie à des informations nouvelles, mais modifie aussi la manière de comprendre et d'exploiter l'information existante ${ }^{49}$.

De manière plus radicale, d'autres auteurs observent que les managers, lors de leurs décisions d'investissement, se bornent souvent à imiter le comportement d'autres managers, à la rigueur sans tenir compte des informations dont ils disposent. Ce comportement se justifie par l'importance de la réputation parmi les valeurs des managers. Ces comportements mimétiques ont été étudiés aussi pour les consommateurs. Deux thèses principales ont été avancées pour expliquer ces comportements apparemment « irrationnels» : les agents se comportent ainsi, soit parce que l'efficience n'est pas leur premier but (en ce cas, il y a d'autres formes de rationalité possible), soit parce qu'il y a des distorsions qui empêchent d'adopter un comportement efficient ${ }^{50}$. Si, dans ce dernier cas, nous restons dans le cadre d'une rationalité limitée substantielle, le premier ouvre au contraire la voie à d'autres formes de rationalité.

On peut par exemple imaginer que, même si la plupart des agents se bornent à imiter les stratégies de ceux qui ont davantage de succès, une certaine inertie persiste tout de même dans le système. En effet, toute modification de stratégie exige des coûts. De ce fait, si l'opportunité de changer se présente plusieurs fois, il devient tout à fait rationnel d'adopter un comportement «myope». Michichiro Kandori, George Mailath et Rafael Rob $^{51}$ adoptent cette interprétation. De ce fait, sur une longue période, les sujets sélectionnent un équilibre favorable au risque. L'inertie structurelle

49. Cohen et Levinthal, 1989.

50. Scharfstein et Stein, 1990; Banerjee, 1992; Bikhchandani, Hirshleifer et Welch, 1992; Lane, 1997; Chamley, 1999.

51. Kandori, Mallath et Rob, 1993. 
au système n'empêche pas l'adoption d'innovations; au contraire, on ne saurait pas avoir celles-ci sans celle-là.

Glenn Ellison et Drew Fudenberg ${ }^{52}$ proposent une interprétation différente. Ils supposent que les sujets modifient leurs décisions d'après les comportements des autres sujets, mais qu'en même temps, leur rationalité soit limitée procédurale. De ce fait, même si, sur la «longue période », on arrive à adopter la meilleure solution possible, cela se fait au prix d'un ralentissement des innovations à court terme. Ce résultat s'explique par la manière dont les informations sont perçues et se propagent. Si une technologie est assez répandue, plusieurs sujets tendent à l'adopter même si une nouvelle technologie apparaît et se révèle plus performante. Par exemple, un fermier peut disposer d'informations sur les techniques des autres fermiers, sans connaître pour autant le rapport entre l'adoption de ces techniques et le revenu. Une situation analogue se présente dans le cas des investissements dans l'éducation des enfants. La perception qu'un sujet (par exemple, un paysan, un fermier) a du rapport entre l'éducation et les avantages économiques et sociaux se forme d'après l'expérience de ses voisins, de ses connaissances, etc. Cependant, cette forme de connaissance empirique peut donner des résultats différents de la réalité si, par exemple, la grande partie des jeunes qui étudient quittent leur village d'origine. Cette approche a le mérite de rendre compte des rigidités des comportements et de la reproduction des conventions à la base des institutions. Pour l'historien, ces aspects sont utiles afin de discuter des phénomènes de longue durée ainsi que de la «mémoire des institutions ». Des perceptions et des conventions constituent un patrimoine commun des sujets qui permet d'aboutir à une solution socialement stable. La rationalité économique éclate grâce à la prise en compte du processus de connaissance lié à l'acquisition des informations; les tensions, voire les superpositions, entre conventions, traditions et innovations permettent de rendre compte des changements, des frictions des temporalités multiples à l'intérieur du même espace chronologique.

Une dernière question se pose encore : si la rationalité est limitée, de quelle manière se transforment les systèmes économiques? Par sélection et élimination ou bien par évolution endogène? De quelle manière ces approches fondées sur l'information économique modifient-elles donc la notion de concurrence?

52. ElLison et FudenBerg, 1993. 


\section{LA SÉLECTION ET L’ÉVOLUTION DES SYSTÈMES ÉCONOMIQUES}

Les études sur l'apprentissage arrivent parfois à faire de la rationalité une variable endogène et à distinguer l'information de la connaissance. Avec quelles implications pour la théorie du développement et pour l'histoire de l'économie?

Une première solution consiste à imaginer que les convictions a priori sont modifiables par l'acquisition des informations et par l'environnement; en ce cas, il n'y a pas de raison d'imaginer des réactions différentes des sujets s'ils sont « rationnels » et s'ils ont tous accès à l'information. Cette homogénéité à l'échelle microéconomique se reproduit au niveau macroéconomique : une fois les «contraintes institutionnelles » éliminées, tous les systèmes suivent la même évolution. L'évolution de la Russie actuelle, qui s'acheminerait vers une économie de marché, peut être prise comme exemple.

Une autre solution est de supposer que les informations ne modifient pas entièrement les préjugés et donc n'affectent pas totalement le comportement des agents. Ces deux approches de l'acquisition de l'information ont trouvé leur correspondance macroéconomique dans l'analyse de la concurrence et de la dynamique des systèmes économiques. On retrouve alors la confrontation entre une approche évolutionniste à la Lamarck, et une approche à la Darwin (par élimination). Cette dernière approche a été envisagée par Jean Tirole selon lequel le sujet économique agit en se disant: «Si j'agis, je donne des informations à la concurrence; il faut donc attendre, en particulier à cause de la baisse du nombre des entreprises, ou bien il faut suivre un apprentissage du type learning by doing ${ }^{53}$. $\gg$ Selon cette approche, les convictions initiales ne comptent guère: elles sont égales pour tous. De ce fait, l'information est cruciale; par contre, elle ne peut jouer ce rôle d'aiguillon que si elle reste privée. La rationalité est instrumentale et, même si elle est limitée, elle peut s'améliorer grâce à l'acquisition des informations. Le système converge vers un sentier d'équilibre et les comportements réels finissent par s'uniformiser selon les modèles prévus par la théorie économique.

L'autre approche (à la Lamarck) a été proposée par Robert Nelson et Sidney Winter ${ }^{54}$. Ils partent du constat que le marché n'élimine pas toujours les entreprises qui n'optimisent pas. Les comportements passés et la routine sont assimilés aux gènes en biologie, tandis que les innovations sont rapprochées des mutations. Le problème devient alors : quel rapport

53. Tirole, 1982.

54. Nelson et Winter, 1982. 
peut-on établir entre l'agent (l'individu, l'entreprise) et le «milieu », le marché? Ces auteurs ont réalisé deux études en prenant comme variable indépendante soit le sujet, soit le « système ». Dans le premier cas, l'évolution de la concurrence oblige les entreprises à modifier leur comportement, ainsi que leur technologie et leur organisation. Au contraire, dans le second cas, l'entreprise ou le sujet change indépendamment des pressions du marché.

La question qui demeure est de savoir comment concilier ces deux points de vue. En effet, si l'on affirme que le comportement du sujet économique dépend de ses convictions initiales et de l'information acquise par la suite, alors on a recours à une rationalité cognitive; autrement, on revient à des formes traditionnelles de rationalité purement instrumentale.

Ce problème a d'importantes implications pour l'histoire de l'économie. Car si l'on suit l'approche à la Darwin, alors les sujets cherchent à minimiser les coûts et ceux qui ne le font pas sont éliminés. De cette manière, on peut donner une valeur cognitive réelle à l'information économique; cependant, celle-ci n'est pas liée à d'autres facteurs sociaux et finit souvent par justifier ex-post des démarches tautologiques. Certains travaux récents sur le processus de connaissance en économie a permis, en partie, de franchir cette difficulté. Les applications historiques de ces démarches mettront alors en évidence l'importance de la concurrence, mais aussi les sauts et les ruptures dans le processus de transformation.

De son côté, l'approche à la Lamarck implique que l'évolution historique de l'économie ne se fasse pas forcément sur la base de l'efficacité économique. Cette solution a le mérite de ne pas isoler l'économie des autres domaines et de mieux distinguer des formes historiques différentes. La mutation est lente et une mémoire demeure dans les comportements des sujets comme des institutions. Le temps est continu. Cependant, cette approche présente aussi une limite forte, à savoir la dichotomie entre la pression du milieu sur le sujet et l'initiative de ce dernier.

Dans un cas comme dans l'autre, nous nous trouvons face à un phénomène majeur: ces dernières années, l'économie politique n'a pas seulement modifié ses instruments et ses modèles, mais aussi ses paradigmes. À l'analogie physique, largement dominante jusqu'à présent, s'ajoute le recours grandissant à l'analogie biologique. Les évolutions des organisations, des marchés et des systèmes économiques sont ainsi discutées en termes de sélection naturelle ou bien d'adaptation. Certains arrivent ainsi à utiliser les modèles d'algorithmes et de jeux élaborés par les biologistes; d'autres parlent des rapports entre les sujets économiques comme d'un réseau de neurones ${ }^{55}$. Les informations (input) sont traitées et transformées avant de donner lieu à des perceptions et à des comportements réactifs.

55. Salmon, 1995. 
Cette orientation nouvelle reflète certes une tendance plus générale, présente dans plusieurs domaines, des sciences humaines aux sciences naturelles jusqu'à la médecine. Dans le cas de l'économie politique, il faudrait comprendre les implications non seulement pour la théorie mais aussi pour les aspects normatifs. Ce problème dépassant les limites de notre investigation, nous pouvons plutôt nous questionner sur l'effet en retour de ces approches nouvelles en économie politique sur l'histoire de l'économie et, en particulier, sur la construction du temps historique.

\section{TEMPORALITÉS HISTORIQUES ET LOGIQUE ÉCONOMIQUE}

Les théories néo-institutionnalistes et le recours à la théorie des jeux ont permis des avancées importantes tant dans la théorie économique qu'en histoire de l'économie. Par rapport aux théories traditionnelles, néo-classiques et marxistes, le «marché » et les institutions, l'État en particulier, sont moins opposés que complémentaires. Des formes organisationnelles différentes sont justifiées sur la base de conventions, d'accords, de perceptions et d'anticipations des sujets. Les représentations du monde économique perdent des sujets sans incertitude ainsi que la recherche à tout prix d'équilibres parfaits. À la place de sujets confrontés au marché, l'accent est surtout mis sur l'interface entre agents.

En histoire de l'économie, ces approches nouvelles ont permis de mieux rendre compte des rigidités, des persistances, des phénomènes de «longue durée ». Les analyses sont davantage fonctionnalistes que nominalistes et s'appuient sur une interaction forte, auparavant quasiment impossible, entre les comportements microéconomiques et les dynamiques macroéconomiques. De ce fait, il a été possible de dépasser des coupures classiques telles que celle entre le «féodalisme » et le « capitalisme », ou celle entre l'économie d'autosubsistance et l'économie de marché. À leur place, on distingue des formes différentes du capitalisme à des époques et dans des lieux différents ou encore un regard nouveau sur des phénomènes tels que le commerce au Moyen Âge, l'évolution des corporations et l'organisation du marché du travail. Ainsi, les problèmes contractuels et les phénomènes de réputation permettent de mieux rendre compte de l'expansion du commerce européen aux $\mathrm{XII}^{\mathrm{e}}$ et $\mathrm{XIII}^{\mathrm{e}}$ siècles que les théories traditionnelles des monopoles. De même, le développement du capitalisme n'est plus lié simplement à la manufacture et à la division du travail, donc aux économies d'échelle; l'accent est par contre mis sur les rapports à l'intérieur de l'entreprise (hiérarchie et contrats).

Finalement, comme il n'y a pas de systèmes socio-économiques figés (le capitalisme, le féodalisme), mais des formes et des relations organisa- 
tionnelles, il n’y a pas de déterminisme historique non plus. Des solutions macroéconomiques différentes sont toutes également envisageables, tout comme des choix et des stratégies multiples sont accessibles à chaque sujet. La notion d'induction en probabilité permet à l'historien de tenir davantage compte des solutions alternatives se présentant tout au long de l'histoire qu'il retrace. Il n'y a ni déterminisme tout court, ni « producteur indépendant », mais un ensemble de désirs, anticipations, conventions et contraintes. Les probabilités ne sont plus employées, comme dans les études économétriques, pour afficher la présence d' «erreurs », de « résidus» non expliqués ou non explicables par l'analyse historique. Au contraire, les probabilités évoquent des choix possibles, des solutions d'attente, même si, effectivement, celles-ci se situent toutes à l'intérieur d'une fourchette déterminée à l'avance.

De ce fait, l'explication causale devient plus souple, car les transformations sont expliquées en termes de probabilités et la comparaison historique ne se fait plus sur une hiérarchie figée, à savoir, le cas anglais comme prototype et les autres solutions expliquées par l'absence de l'un ou de l'autre facteur. Au contraire, des traditions et des conditions différentes peuvent légitimer des solutions différentes mais également efficaces.

Cependant, à côté des progrès, ces approches présentent aussi des limites importantes. Pour commencer, les coûts de transaction demeurent une boîte noire; de ce fait, l'explication historique est souvent tautologique. D'autre part, si les théories néo-institutionnalistes et les théories des jeux arrivent à rendre compte des persistances et des continuités, elles ont par contre du mal à expliquer les ruptures historiques. Et même dans une optique de continuité et de changements lents, il est possible de justifier la mise en place de l'une ou de l'autre forme d'organisation, tandis que l'explication de son déclin est souvent faible. De ce fait, peut-on distinguer le(s) capitalisme(s) seulement par ses(leurs) formes organisationnelles? Les théories néo-institutionnalistes et les jeux trouvent des applications d'autant plus différenciées dans l'espace et dans le temps que les aspects envisagés sont peu nombreux. Mieux, il y a la tentative explicite de ramener les facteurs anthropologiques et sociologiques aux seules variables économiques. L'éthique économique médiévale, les règles des corporations, le système de l'annone et les entreprises japonaises s'expliquent tous par les seules exigences de minimiser les coûts de transaction.

Ces difficultés dans l'explication macrohistorique se conjuguent d'ailleurs avec celles dans la description microéconomique. La théorie des contrats à la base des jeux présuppose des sujets ayant des stratégies et des objectifs différents, mais en tout cas de force égale. L'autorité n'est pas synonyme de pouvoir et la hiérarchie n'est pas contraignante. De ce fait, les rapports dans une organisation (une entreprise, une famille, un bureau) 
s'expliquent et sont censés se modifier exclusivement sur la base des notions de contrainte, motivation et punition. C'est aussi pourquoi les stratégies peuvent être certes différentes, mais la rationalité demeure instrumentale et ne se modifie pas pendant le déroulement du jeu.

À partir de là, ces approches atteignent deux limites. En premier lieu, elles ne tiennent pas compte du fait que l'information (économique) n'est pas forcément produite dans un but économique (l'efficacité), elle peut l'être aussi pour des raisons de prestige, de pouvoir, etc. En outre, l'emploi de l'information ne s'accorde pas forcément avec une rationalité instrumentale. C'est pourquoi la rationalité peut être limitée non seulement parce que les informations sont insuffisantes, mais aussi parce que les perceptions du sujet, la manière dont il conçoit et transforme les signaux sont différentes et surtout ne sont pas influencées que par le calcul économique. Que l'on songe, à ce propos, à la production des archives soviétiques ou aux cartulaires médiévaux. Dans un cas comme dans l'autre, la production d'information n'est pas forcément suivie de son emploi rationnel du point de vue de l'économie, mais sert plutôt à légitimer l'institution ou le sujet qui la produit. Cette considération permet de dégager le terrain d'un faux débat dans la théorie de l'information appliquée à l'histoire, à savoir : la concentration et la production d'information répondent-elles à des soucis de monopole ou bien d'efficacité ? La question présuppose une production et une gestion de l'information purement instrumentale, c'est-à-dire orientée vers l'efficacité.

En second lieu, en tenant compte des motivations non économiques dans la production et dans la gestion de l'information, il est possible de s'interroger sur la place réelle que les variables économiques stricto sensu ont à l'une et à l'autre époque, pour l'un ou l'autre des sujets envisagés. Certains travaux récents sortent du cadre d'une rationalité purement instrumentale et tiennent compte du processus de connaissance. Ils ont permis de rendre la rationalité et les stratégies économiques des variables endogènes. Ralentissements et innovations sont étroitement liés et les mémoires hétérogènes des sujets finissent par affecter leurs perceptions et leurs anticipations, donc l'évolution de leur comportement. L'économie spatiale devient une économie des temps éclatés, multiples et pourtant unis entre eux.

Dans quelle mesure l'historien peut-il avoir recours à ces approches?

Tout d'abord, il faut écarter une solution trop souvent avancée par les historiens et par plusieurs courants d'économistes, à savoir rapprocher davantage les modèles et les schémas théoriques de la «réalité ». Le «manque de réalisme » a été l'une des critiques les plus répandues adressées à la théorie néo-classique traditionnelle et, en particulier, à ses hypothèses de concurrence totale, de connaissance parfaite et de manque d'incertitude. Ces mêmes critiques sont aujourd'hui dirigées contre la théo- 
rie des jeux. Deux réactions principales se dégagent. Un courant, minoritaire, estime que la théorie économique ne doit pas forcément correspondre à la réalité, car elle peut et doit être utilisée comme modèle heuristique.

Cette solution a été cependant critiquée par les « réalistes », au nom de l'importance normative de l'économie : la théorie sert à orienter les politiques économiques et, de ce fait, elle doit être réaliste. On essaye alors de mieux rapprocher les modèles de la « réalité ». C'est à partir de cette prémisse que ce sont développées les approches néo-institutionnalistes, l'analyse du consommateur, la théorie de l'oligopole, jusqu'à une partie de la théorie des jeux. Un corollaire important de cette approche est que, afin d'être réaliste, la théorie économique doit avoir recours à l'histoire. «L'histoire est la seule méthode qui permet d'explorer directement le spectre complet de la réalité »; donc «l'histoire économique est le laboratoire de l'économie théorique ${ }^{56}$. Même si de telles affirmations ne manqueraient pas de flatter certains historiens, nous pouvons nous demander ce qu'on entend par « histoire».

Un des théoriciens de l'économie néo-classique ainsi revisitée, Frank Hahn, définit l'histoire comme «le processus dynamique par lequel les agents économiques passent de leur état d'ignorance à une connaissance qui permet d'atteindre une position d'équilibre ${ }^{57} \gg$. La connaissance (et l'information économique, en particulier) est donc conçue exclusivement comme un processus visant à atteindre un comportement optimisant, en quelque sorte préexistant à la connaissance elle-même. Le post hoc ergo propter hoc non seulement n'est pas écarté, mais il devient au contraire la règle explicative dominante, voire unique. Cette tendance positiviste forte oppose un temps réel, historique, au temps logique de l'économie ${ }^{58}$.

Il nous paraît donc plus pertinent d'adhérer à l'opinion de ceux qui estiment que ce n'est pas en s'appuyant sur le « réalisme » des modèles que l'économie théorique et l'histoire de l'économie pourront sortir, l'une du cadre de la théorie néo-classique et l'autre du problème entre théorie économique et analyse historique, entre temps historique(s) et temps logique(s) ${ }^{59}$.

Il faudra alors que le recours aux schémas économiques en histoire ne dépasse pas un cadre heuristique. Ce qui équivaut à éviter des notions passe-partout, telles que celles de marché, d'institution, ou encore de coûts de transaction et d'information. L'emploi le plus souvent a-temporel que l'on fait de ces catégories a donné l'illusion de comparaisons efficaces et d'interprétations puissantes. Qu'il s'agisse de l'Angleterre au XvI ${ }^{\mathrm{e}}$ siècle, de

56. SNooks, 1993, p. 16.

57. HAHN, 1987.

58. SNOOKS, 1993, p. 25.

59. Hodgson, 1988. 
l'Inde au XVIII 'ou de la Russie en 1993, le «marché » ou l' «économie de marché » ont été mis en cause. Le résultat a été le plus souvent contraire aux attentes : on a décrit le fonctionnement d'un système qui, en dépit de l'accent mis sur l'évolution historique, reste identique à lui-même.

Il faudra aussi éviter que la notion d' " information » devienne une nouvelle boîte noire. L'information n'a pas d' "utilité objective», pas plus qu'elle n'est traitée passivement par les sujets envisagés. Il serait important de se questionner sur sa genèse, son emploi et sa signification (socioéconomique et épistémologique) dans le cas historique étudié. Cela, tout en sachant que le langage et les catégories préexistent à l'information ellemême. L'analyse de l'évolution historique des termes utilisés et celle des catégories statistiques, économiques, démographiques, etc. constituent alors un préalable à toute démarche de ce genre.

Il faudra également comparer l'évolution de ces constructions «savantes» à celle des institutions, des groupes sociaux et des individus concernés ${ }^{60}$. Il n'y a pas une « réalité » à opposer aux perceptions mais une interaction forte, moins logique que constitutive. Cette solution peut offrir une issue à l'histoire du développement économique qui, longtemps contrainte par l'analogie physique, risque aujourd'hui de se modeler dans l'analogie biologique. Ce virage épistémologique s'accompagne du retour à des sentiers de développement prédéterminés que, selon des modalités différentes, les approches anthropologiques et la théorie des jeux avaient contribué à balayer. Plutôt que de miser sur un retour à l'analogie biologique, l'histoire devrait s'interroger sur les (auto)perceptions des temps biologiques des sujets envisagés et de leurs superpositions et décalages éventuels avec les pulsations des temps historiques propres aux individus, aux groupes sociaux différents, à des moments donnés. Par exemple, les perceptions du vieillissement influencent celles du temps et donc les choix des individus tout comme les représentations collectives. Les décisions au sujet de l'épargne et de l'investissement seront prises en fonction de ces conditions.

De manière générale, l'histoire de l'économie ne pourra se passer des reconstructions subjectives du temps. Les sujets et les institutions ont leurs propres mémoires, qui se modifient dans le temps et avec elles leurs perceptions et leurs anticipations. Ce qui a été perçu comme rationnel il y a un an, ne l'est plus aujourd'hui, soit parce que les conditions extérieures se sont modifiées, soit parce que les évaluations et les jugements du sujet se sont aussi modifiés ${ }^{61}$. C'est pourquoi, il faudra distinguer, à des moments

60. Pour des démarches dans ce sens, voir Stanziani, 1992 et 1998.

61. Sur ces aspects, voir SHACKLE, 1954. 
différents, les processus d'apprentissage, c'est-à-dire la manière dont un sujet économique acquiert et traite l'information. Cette démarche, bien entendu, se transforme dans le cas d'une institution ou d'une organisation, en un examen des perceptions différentes de la même information à l'intérieur de la même organisation. Par exemple, le service technique et celui du marketing partagent-ils la même notion de «qualité » d'un produit? De plus, cette approche peut offrir une solution à un problème épistémologique apparemment sans issue : comment concevoir les «solutions possibles » envisageables par le sujet?

La théorie des jeux offre là une suggestion intéressante : le choix est toujours évoqué et l'histoire naît de bifurcations continuelles. Ce qui permet de dépasser l'alternative traditionnelle entre un libre choix et des contraintes extérieures, si longtemps débattue en historiographie, en sociologie et dans les sciences politiques. Cependant, cette acquisition de la théorie des jeux trouve aussi une limite majeure : le processus du choix est, lui, en dehors du temps.

Cette impasse épistémologique peut éventuellement connaître une solution dans l'intégration des choix réitérés, propres à la théorie des jeux, dans une analyse des perceptions subjectives du choix, selon les approches des études récentes sur le processus de connaissance. De son côté, l'historien devrait inclure ces perceptions subjectives (la rationalité limitée procédurale) dans leur temporalité. La manière dont chaque sujet ou institution envisage le rapport entre libre choix et contraintes extérieures se modifie au fil du temps. Analyser cette évolution sera donc préalable à toute analyse historique des « libertés» et des «contraintes » « réelles».

De ce fait, un événement imprévu ne s'inscrit plus dans l'ancien débat sur le rôle du hasard en histoire, mais il sert plutôt à mettre en évidence le fait qu'un événement conditionne les choix individuels non seulement parce qu'il modifie l'horizon d'attente et les anticipations, mais aussi parce qu'il provoque un examen nouveau des événements passés, qui conditionne à son tour les choix à venir. Ces aspects offrent une grille de lecture qui est à la fois assez efficace comme instrument heuristique et assez souple pour être employée dans des contextes historiques et spatiaux différents sans renoncer pour autant à une notion forte des temporalités historiques.

Alessandro StanZiani (novembre 1999). 


\section{LISTE DES RÉFÉRENCES}

Abreu (Dilip), 1988, "On the theory of infinitely repeated games with discounting », Econometrica, vol. LVI, 2, mars, p. 383-396.

Abreu (Dilip), Milgrom (Paul) et Pearce (David), 1991, «Information and timing in repeated partnership », Econometrica, vol. LIX, nov., p. 1713-1733.

Arrow (Kenneth) et HonKapohja (Sappo), 1985, Frontiers of economics, Oxford, Oxford University Press.

Aumann (Robert), 1985, «What is game theory trying to accomplish », in ARrow et HONKAPOHJA, 1985, p. 39.

BANERJEE (Abhijit), 1992, " A simple model of herd behaviour », Quarterly Journal of Economics, vol. CVII, 3, august, p. 797-817.

BERG (Maxime), 1991, « On the origin of capitalist hierarchy », in Gustafsson, 1991, p. 173-194.

Bikhchandani (Sushil), Hirshleifer (David) et Welch (Ivo), 1992, «A theory of fads, custom and cultural change as informational cascades », Journal of Political Economy, vol. C, 5, p. 992-1026.

BOYER (George), 1990, An economic history of the English poor law, 1750-1850, Cambridge, Cambridge University Press.

Carlos (Ann) et Nicholas (Stephen), 1988, «Giants of an earlier capitalism. The early chartered trading companies as modern multinationals », Business History Review, vol. LXII, automne, p. 398-419.

Chamley (Christophe), 1999, «Coordinating regime switches », Quarterly Journal of Economics, vol. CXIV, 3, p. 869-905.

Conen (Wesley) et Levinthal (Daniel), 1989, «Innovation and learning. The two faces of R. and D. », Economic Journal, vol. XCIX, p. 569-596.

Demsetz (Harold), 1988, Ownership, control and the firm. The organization of economic activity, Oxford, New York, Oxford University Press, vol. I.

Elbaum (Bernard), 1989, «Why apprenticeship persisted in Britain but not in the United States », Journal of Economic History, vol. XLIX, 2, p. 337-349.

Elbaum (Bernard) et Lazonick (Walter), 1986, The Decline of the British economy, Oxford, Oxford University Press.

Ellison (Glenn) et Fudenberg (Drew), 1993, « Rules of thumb for social learning », Journal of Political Economy, vol. CI, 4, p. 612-643.

Fenoaltea (Stefano), 1976, « Risk, transaction costs and the organization of medieval agriculture », Explorations in Economic History, vol. XIII, p. 129-151.

Fenoaltea (S.), 1988, "Transaction costs, whig history and the common fields », Politics and Society, vol. XVI, p. 171-240, repr. in Gustafsson, 1991, p. 107172.

Fogel (Dan), 1992, «Second thoughts on the European escape from hunger. Famines, chronic malnutrition and mortality rates », in ID., Nutrition and poverty, Oxford, Clarendon Press, p. 243-286.

Fudenberg (Drew) et Tirole (Jean), 1991, Game theory, Cambridge, Cambridge University Press. 
GibBons (Robert), 1992, A primer in game theory, Englewood Cliffs, NJ, Prentice Hall.

Gomez (Pierre-Yves), 1994, Qualité et théorie des conventions, Paris, Économica.

GreIF (Avner), 1989, « Reputation and coalition in medieval trade. Evidence on the Maghribi traders », Journal of Economic History, vol. XLIX, 4, p. 857-882.

GreIF (A.), 1993, «Contract enforceability and economic institutions in early trade. The Maghribi traders' coalition », American Economic Review, vol. LXXXIII, 3, p. 525-548.

GREIF (A.), 1998, « Théorie des jeux et analyse historique des institutions. Les institutions économiques au Moyen Âge », Annales. HES, vol. LIII, 3, p. 597-634.

Greif (Avner), Milgrom (Paul) et Weingast (Barry), 1994, « Coordination, commitment and enforcement. The case of the merchant Guild », Journal of Political Economy, vol. CII, 4, p. 745-776.

Gustafsson (Bo), 1991, Power and economic institutions. Reinterpretations in economic history, Northampton, MA, Edward Elgar.

HaHN (Frank), 1987, « Information, dynamics and equilibrium », Scottish Journal of Political Economy, vol. XXXIV, p. 321-334.

Hamilton (Gillian), 1996, «The market for montreal apprentices. Contract length and information », Explorations in Economic History, vol. XXXIII, 4, p. 496523.

Hargreaves Heap (Shaun), 1989, Rationality in economics, Oxford, Oxford University Press.

Harsanyi (John), 1967, « Games with incomplete information played by "Bayesian" players », Management Science, vol. XIV, 3, p. 159-182.

Hodgson (Geoffrey), 1988, Economic and institutions, Cambridge, Cambridge University Press.

Jones (S. R. H.) et VILle (Simon), 1996, «Efficient transactors or rent-seeking monopolists? The rationale for early chartered trading companies », Journal of Economic History, vol. LVI, 4, p. 898-915.

Kandori (Michichiro), Mailath (George) et Rob (Rafael), 1993, «Learning, mutation and long run equilibria in games », Econometrica, vol. LXI, 1, p. 29-56.

KHAN (Zarina), 1995, « Property rights and patent litigation in early 19th-century America », Journal of Economic History, vol. LV, 1, p. 58-97.

Kiefer (Nicholas) et Nyarko (Yaw), 1995, "Savage-Bayesian models of economics », in Kirman et SaLmon, 1995.

KIESLING (Lynne), 1996, «Institutional choice matter. The poor law and implicit labor contracts in Victorian Lancashire », Explorations in Economic History, vol. XXXIII, 1, p. 65-85.

Kirman (Alan) et SALmon (Mark), 1995, Learning and rationality in economics, Oxford, Oxford University Press, p. 40-62.

KNIGHT (Frank), 1964, Profit, risk and uncertainty, original de 1921, New York, A. Kelly.

Komlos (John) et LANDES (Richard), 1991, « Anachronistic economics. Grain storage in medieval England », Economic History Review, vol. XLIV, 1, p. 36-45.

KREPS (David) et WILSON (Robert), 1982, «Sequential equilibria », Econometrica, vol. L, 4, p. 863-894.

LANE (David), 1997, «Is what good for each best for all? Learning from others in the information contagion model », in Brian ArThur, Steven Durlauf et David Lane, The Economy as an evolving complex system, Reading, MA, Perseus Books, vol. II, p. 105-127.

LAZONICK (Walter), 1990, Business organization and the myth of market economy, Cambridge, Cambridge University Press. 
LAzonick (W.), 1991, «Organization and markets in capitalist development », in GustafsSON, 1991, p. 253-301.

McCloskey (Donald), 1976, «English open fields as behaviour toward risk », in Paul Uselding, éd., Researches in economic history, Greenwich, CT, JAI Press, vol. I, p. 124-171.

McCloskey (D.), 1991, "Conditional economic history. Reply to Komlos and Landes », Economic History Review, vol. XLIV, 1, p. 128-132.

McCloskey (Donald) et NASH (John), 1984, « Corn at interest. The extent and cost of grain storage in medieval England», American Economic Review, vol. LXXIV, 1, p. 174-187.

Milgrom (Paul) et RoberTs (John), 1992, Economics, organization and management, Englewood Cliffs, NJ, Prentice Hall.

NASH (John), 1951, « Non-cooperative games », Annals of Mathematics, vol. LIV, 2, p. 286-295.

NeLSON (Robert) et WinTER (Sidney), 1982, An evolutionary theory of economic change, Cambridge, Cambridge University Press.

Nielsen (Randall), 1997, «Storage and English government intervention in early modern grain markets », Journal of Economic History, vol. LVII, 1, p. 1-33.

North (Douglass), 1981, Structure and change in economic history, New York, NY, W. W. Norton.

North (Douglass) et Thomas (Robert), 1973, The Rise of Western civilization. A new economic history, Cambridge, Cambridge University Press.

North (Douglass) et Weingast (Barry), 1989, «Constitution and commitment. The evolution of institution governing public choice in 17th-century England », Journal of Economic History, vol. XLIX, 4, p. 803-832.

Polanyi (Karl), 1983, La Grande Transformation, $1^{\text {re }}$ éd. angl. 1944, ici trad. franç. Paris, Gallimard.

Postel-Vinay (Gilles), 1998, La Terre et l'argent. L'agriculture et le crédit en France du XVIII ${ }^{e}$ au début du Xx siècle, Paris, Albin Michel (L'Évolution de l'humanité).

Revue économique, 1989, numéro spécial, 2, «L'économie des conventions ».

Rosenthal (Jean-Laurent), 1992, The Fruits of Revolution, Cambridge, Cambridge University Press.

Rosenthal (J.-L.), 1994, «Rural credit market and aggregate shocks. The experience of Nuits-Saint-Georges, 1756-1776 », Journal of Economic History, vol. LIV, 2, p. 288-306.

SALmon (Mark), 1995, « Bounded rationality and learning. Procedural learning », in KiRMAn et SALmON, 1995, p. 236-275.

Scharfstein (David) et Stein (Jeremy), 1990, « Herd behaviour and investment », American Economic Review, vol. LXXX, 3, p. 465-479.

Schelling (Thomas), 1977, The Strategy of conflict, Oxford, Oxford University Press.

Selten (Reinhard), 1978, "The chain store paradox », Theory and Decision, 9, p. 127-159.

ShackLE (George L.), 1954, "The complexe nature of time as a concept in economics », Economia internazionale, vol. VII, 4, p. 743-757, repr. in ID, Time, expectations and uncertainty in economics, Northampton, MA, Edward Elgar, 1990, p. 3-14.

Simon (Herbert), 1982, Models of bounded rationality, Boston, MIT.

Snooks (Gary), 1993, Economics without time, Londres, Routdlege. 
SoKolofF (Kenneth), 1988, «Inventive activity in early industrial America. Evidence from patent records, 1790-1846», Journal of Economic History, vol. XLVIII, 4, p. 813-850.

Stanziani (Alessandro), 1992, "Les statistiques des récoltes en Russie, 1905$1928 »$, Histoire et mesure, vol. VII, 1-2, p. 73-98.

Stanziani (A.), 1998, L'Économie en révolution. Le cas russe, 1870-1930, Paris, Albin Michel (L'Évolution de l'humanité).

STIGLeR (George), 1968, The Organization of industry, Boston, MIT.

STIGLER (G.), 1982, The Economist as a preacher, Boston, MIT.

Tirole (Jean), 1982, On the possibility of speculation under rational expectations, Cambridge, Cambridge University Press.

Williamson (Oliver), 1985, The Economic Institutions of capitalism, New York, The Free Press. 